\title{
Measuring the Effectiveness of User Interventions in Improving the Seated Posture of Computer Users
}

\author{
Paul Duffy ${ }^{1}$ and Alan F. Smeaton ${ }^{1,2}$ \\ ${ }^{1}$ School of Computing and ${ }^{2}$ INSIGHT: Data and Analytics Research Centre \\ Dublin City University, Glasnevin, Dublin 9, Ireland. \\ alan.smeaton@dcu.ie
}

\begin{abstract}
Extended periods of time sitting in front of a computer give rise to risks of developing musculoskeletal disorders. In the workplace, computer use contributes considerably to employee injury and results in significant costs to the employer in terms of sick leave and injury claims. Due to these risks there has been significant research into the areas of posture classification and subject intervention to improve posture in an office environment. The Kinect ${ }^{\mathrm{TM}}$ has been shown to be a suitable hardware platform for posture classification. This paper presents a system for posture classification and novel subject intervention that leverages each of three distinct forms of persuasive computing and explores the success of each type. Our results show significant improvement in posture results from the most effective of our intervention types.
\end{abstract}

\section{Introduction}

It is known that poor ergonomic posture during computer use is a risk factor in developing musculoskeletal disorders and as a result, approximately one third of lost-day cases in the US workplace have been attributed to musculoskeletal disorders [3]. A key contributor to the prevalence of such disorders in the office workplace is the use of computers for prolonged periods of time [6]. A variety of ergonomic systems have been proposed, several of them now available as commercial products. The common thread across such systems is to detect an undesired behavior that is known to increase the risk developing musculoskeletal symptoms and to provide a user intervention to attempt to change behavior.

Persuasive technologies [5] are an emergent trend in human-computer interaction and are a means to influence subjects to perform or adopt to a chosen behavior. The work reported in this paper explores the idea that an ergonomic system to monitor and correct posture will be more effective if its design is approached with the concepts of persuasive technology at its core. 


\section{Background}

\subsection{Existing methods of posture classification}

Evidence exists that there is a relationship between seated posture in the workplace and musculoskeletal symptoms such as neck and back pain [6]. Research has shown that the increase in daily computer usage results in a greater likelihood of symptom reporting [1]. During the study it was shown that exceeding 3 hours of continuous computer usage led to a $50 \%$ higher likelihood of reporting musculoskeletal discomfort.

Several systems for monitoring posture exist, one of which is manual posture assessment by means of observation. In this method, a trained ergonomist carries out an observation-based assessment of a participant and classifies the person's movement against set posture scales. A study [9] was carried out into the accuracy of such observational measurements where observers were required to classify a participant's elbow and shoulder posture based on a three value scale $<40$, [40-80], or $>80$. Results showed an average probability of misclassification of $30.1 \%$. This highlights the need for better tools which can classifying posture, automatically or at least which can assist a manual classification.

Another example system provides an holistic solution for posture monitoring [8] with real time feedback and summarisation of a person's postures throughout the day. This system uses a video camera and microphone placed on top of the participant's computer monitor to ambiently log the subject's activities. Based on classifications the subject can be alerted through an on-screen dialog and can also choose to review a summary of the time spent sitting in different postures over the course of the day. Other posture classification approaches based on accelerometers have not been as successful as camera-based.

Research into the use of the Kinect ${ }^{\mathrm{TM}}$ into analysis of postural control and balance has shown that it is a device capable of making classifications of a person's joint movements with high accuracy [2]. The Kinect ${ }^{\mathrm{TM}}$ was favourably benchmarked against a more commonly-used 3D camera motion capture system made up of several video cameras and 19 markers placed on the body.

\subsection{Influencing subjects to change behaviour}

Research has been carried out on how best to interrupt a user from their everyday office work in order to inform them of their poor posture, and to do so with the smallest impact to their workflow and productivity [7]. The three options that were proposed were graphical feedback, physical feedback and haptic feedback. Graphical feedback was in the form of a popup window on the subject's desktop while physical feedback was in the form of a toy flower placed on the subject's desk, connected to a USB interface that would mimic the subject's posture by bending its leaves and stem. The haptic feedback was provided using the vibrations from a game console controller. A pilot was run and the performance of each intervention method was assessed where subjects were surveyed to gather information on how disruptive each medium was. Results favoured the physical 
feedback approach as the haptic feedback was considered too disruptive by a considerable number of the pilot's participants. It was also found that subjects were more likely to ignore or postpone graphical, compared to physical alerts.

Using computers as a persuasive technology has been described as any interactive technology that attempts to change a person's behaviour [5]. This can motivate behaviour change by providing experiences and sensations that create a cause and effect relationship between the person and the computer. One researcher proposes an 8-step system for successful design of persuasive technologies [4] which include the selection of a simple behaviour to target, designing for a receptive audience, identifying what is currently preventing the behaviour and choosing an appropriate technology channel for communications. In the case of our study we are targeting poor posture and will attempt to use three different technology channels to trigger behavior change.

\section{$3 \quad$ Experimental Methods}

\subsection{Units of measurement}

In designing a posture classification system we need to decide on what measurements yield a good approximation of seated posture, enough to differentiate between good, and bad. The Kinect ${ }^{\mathrm{TM}}$ is capable of tracking torso, limb and head movements, however the nature of tracking a subject seated at a desk with the Kinect ${ }^{\mathrm{TM}}$ mounted on the computer screen means that the line of sight may be blocked by objects in the environment. We know the subject's head is the least-occluded body part and we believe that measuring its angle, relative to the plane of the sensor, could provide a valid and usable approximation of overall posture.

An experiment was setup to validate what body parts can be reliably tracked using the Kinect ${ }^{\mathrm{TM}}$ while a subject is seated in an office cubicle, operating a desktop computer. Software was written to log the tracking state of each of the subject's joints. In total the Kinect ${ }^{\mathrm{TM}}$ sensor is able to track up to 20 joints of a subject as shown in Figure 1. Using the Kinect ${ }^{\mathrm{TM}}$ SDK we can create a skeleton data stream that provides information on each of our subjects' 20 joints. This information includes joint position in $3 \mathrm{D}$ space, joint angle and a state variable indicating the type of tracking on the joint. The state of each joint can be Tracked, Not Tracked or Inferred, the later being a joint whose position is determined indirectly, based on its connecting joints. For this experiment we discarded inferred data from the data stream and just logged those joints in a Tracked state. Data was gathered at the rate of one frame per second over a period of 4 hours, and the state of each joint was logged for each frame.

The subject remained seated for 4 hours and performed their normal office working routine, moving around the chair, twisting and turning to reach things, answer the phone, etc., and generally reflecting the regular, dynamic movements which are characteristic of good seating behaviour. The logged data was analysed and the result presented in Table 1. Joints not listed had 0\% detection. Skeletal 


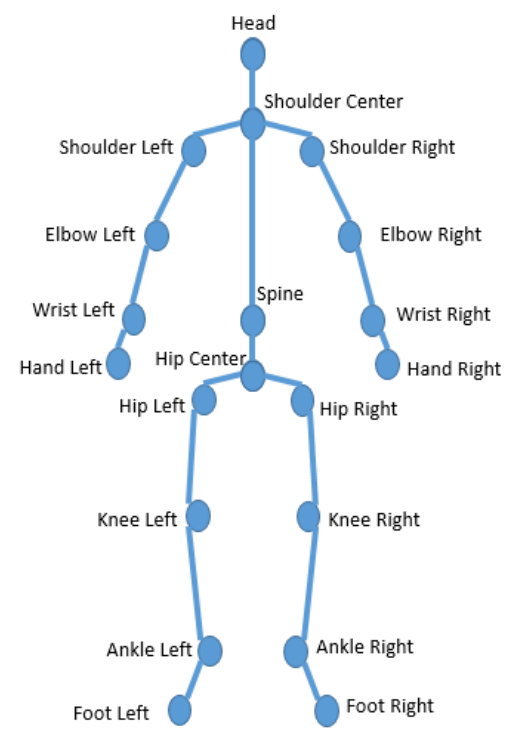

Fig. 1. Kinect ${ }^{\mathrm{TM}} 20$ point skeleton

Table 1. \% time each joint was successfully tracked over a 4-hour period.

\begin{tabular}{|c|c|}
\hline Joint & \% Time \\
\hline Head & $99.98 \%$ \\
Right Shoulder & $87.83 \%$ \\
Right Elbow & $70.56 \%$ \\
Right Wrist & $68.42 \%$ \\
Right Hand & $68.08 \%$ \\
\hline
\end{tabular}

\begin{tabular}{|c|c|}
\hline Joint & \% Time \\
\hline Shoulder Centre & $99.08 \%$ \\
Left Shoulder & $89.65 \%$ \\
Left Elbow & $58.52 \%$ \\
Left Wrist & $56.62 \%$ \\
Left Hand & $55.98 \%$ \\
\hline
\end{tabular}

tracking was most successful on the subject's head, directly in front of the sensor and not in contact with any objects from the environment. It is possible that the raised back on the subject's chair interfered with the tracking of the shoulder joints. Tracking of the subject's right elbow, wrist and hand was more successful than tracking the subject's left side. We noted that the subject used the mouse with their right hand so their arm was resting out to the side of their body for much of the experiment. The sensor was unable to track the position of the subject's spine, hips, knees, ankles or feet as expected. Based on this, we use motion tracking of subjects' head as the basis for posture classification.

\subsection{Classifying Posture}

This experiment involved measuring the sensor's response to changes in the seated position of 3 subjects who were simulating various types of poor posture. Firstly a reference measurement was taken of each subject sitting in a comfortable upright position. Next they moved between four poses, sitting forward with 
their back hunched over, leaning back on their chair, resting heavily on their left arm rest and then on their right arm rest. During each pose a measurement was taken from the sensor.

The experiment was carried out twice for each of three subjects, once in a well-lit room and again in a poorly-lit room. This allowed us to validate the sensor's ability to measure posture on a range of people and in different lighting conditions. A camera was setup on a tripod to document each pose with photos taken at the same time as each measurement. The results $t$ are shown in Table 2, where values are expressed in terms of the difference in the angle of the subject's head compared to the reference measurement for each pose.

Table 2. Delta in degrees from resting position

\begin{tabular}{|c|c|c|c|c|c|}
\hline Position & Axis & Subject A & Subject B & Subject C & Lighting \\
\hline Lean Fwd & $\mathrm{Z}$ & 11 & 8 & 14 & Bright \\
Lean Fwd & $\mathrm{Z}$ & 8 & 10 & 13 & Dim \\
\hline Lean Back & $\mathrm{Z}$ & 2 & 2 & 3 & Bright \\
Lean Back & $\mathrm{Z}$ & 3 & 5 & 6 & Dim \\
\hline Lean Left & $\mathrm{X}$ & 13 & 11 & 14 & Bright \\
Lean Left & $\mathrm{X}$ & 11 & 11 & 10 & Dim \\
\hline Lean Right & $\mathrm{X}$ & 16 & 14 & 14 & Bright \\
Lean Right & $\mathrm{X}$ & 12 & 15 & 11 & Dim \\
\hline
\end{tabular}

Our analysis reveals that estimating posture based on head position is least sensitive to leaning backwards and that change in lighting had little effect on results. Our system now defines any posture that results in a delta of +10 degrees on either $\mathrm{X}$ or $\mathrm{Z}$ axis to be considered as bad posture.

\subsection{Implementation of the posture classifier}

Our posture classifier was implemented in C\# .Net using the Kinect ${ }^{\mathrm{TM}}$ developer toolkit and it models the subject's posture as a state machine with 4 possible states, Unknown, Good, Z Bad and X Bad. Unknown represents the subject's posture when tracking is not possible, for example when the subject is away from the desk or computer or is standing at the desk. Good posture is any posture where the subject's head stays within 10 degrees of their reference position. Z Bad is a state to represent forward leaning or slouching by the subject. A state of X Bad indicates the subject is leaning to either their left or right sides. The state machine raises events to the application layer and allows us to deliver interventions to the subject.

The software only uses the $3 \mathrm{D}$ depth sensor components of the Kinect ${ }^{\mathrm{TM}}$ meaning it can operate with the built-in RGB camera and microphone disabled. As such, it offers a viable option for posture classification in a environment where privacy is necessary, such as an office environment. The information gathered on each subject who used our posture intervention system was limited to just the angle as measured by the sensor of their head on both the $\mathrm{X}$ and $\mathrm{Z}$ axes, captured once per second throughout each session. 


\subsection{Intervention Design}

We used three methods of delivering interventions, the first of which controls the brightness of the monitor. Whenever the subject's posture moves into a bad state for more than a threshold period of time, the intervention triggers and the screen dims. Once the subject corrects their posture the screen returns to normal brightness. This method was intended to create a cause-effect relationship between the subjects and the computer and to train the subject into knowing that if they keep sitting up straight they will not be interrupted yet when the intervention does happen the subject can still see the screen and continue working without interruption if the subject is at a critical phase of work.

The second intervention is purely information based. It consists of a popup window that is displayed to the subject once per hour. A dialog window displays a summary showing the number of minutes spent sitting with good posture, the number of minutes sitting leaning forward and the number of minutes leaning to one side. Again, the subject can choose to ignore the intervention and the intervention was delivered once per hour, regardless of how good the subject's posture was during that time period. The final intervention was also delivered as a popup message with a set of encouraging messages which provide positive re-enforcement to the subject when they use good posture. In general, because these latter two interventions occur once per hour we expect them to be less effective than the real-time response of the monitor brightness change.

\subsection{User Trials}

Having defined and developed the software for our three intervention types, the next phase was to trial each method with subjects to anyalyse what effect each had on their posture. We selected 4 subjects to take part in the trial and each subject was required to use the software for four days. On each day of the three interventions was used, with the fourth day being a day with no interventions at all. Reserving one day per subject to just record posture and not intervene allows us to gather information on how much each person normally corrects their posture and gauge the impact of each intervention.

Each subject's posture throughout each day was recorded by the sensor and logged for analysis. In order to ensure there were no biasing effects from the order in which each intervention was delivered, a Latin square's approach was used to provide a different ordering of interventions for each subject.

In order to facilitate this experimental approach, each subject was given a set of daily activation keys and instructions on which day to use each key. When the application loaded up they were prompted to enter a key which in turn selected the form of intervention they would be receive for that day. After completing a short sensor calibration exercise the software began monitoring their posture and delivering interventions as appropriate. At the end of a four-hour session the subject simply exited the application and logging was stopped. 


\section{Results}

\subsection{Overall Posture}

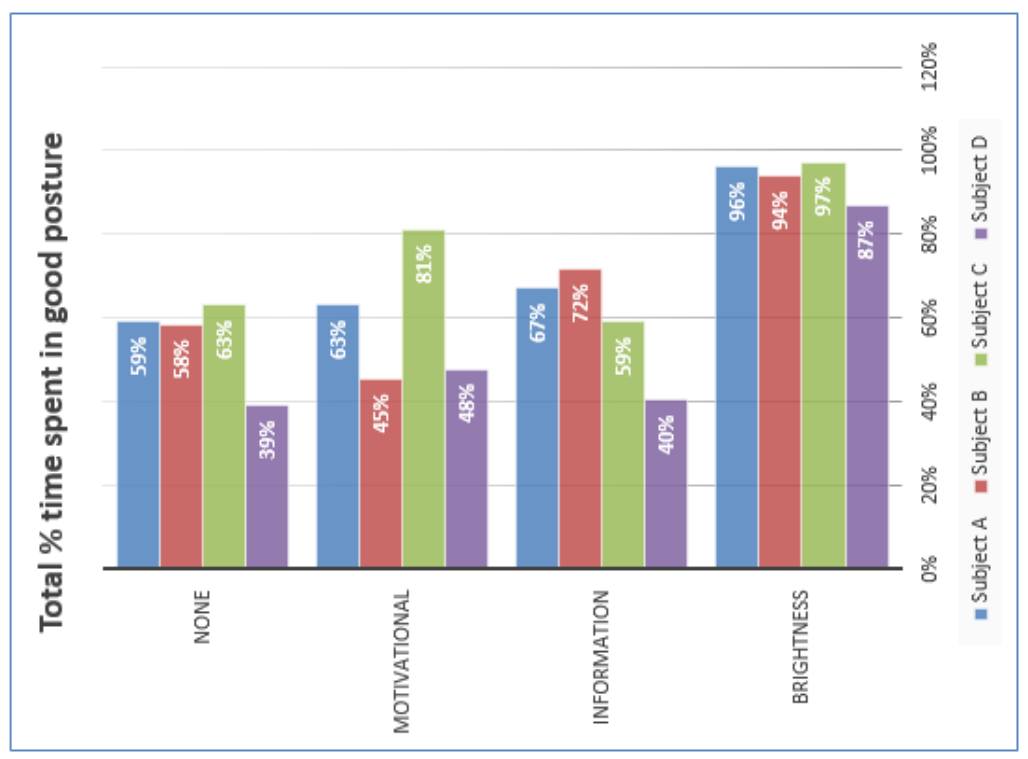

Fig. 2. Percentage time spent in good posture by subject and intervention type.

The first thing we did was to extract the total amount of time spent sitting in a good posture per subject for each intervention type they received, and this information is graphed in Figure 2. For our motivational intervention we see that all four subjects showed improved posture over their day with no intervention at all. For the information-based intervention we see that two subjects showed notable improvement in their posture, however Subject $\mathrm{C}$ showed poorer posture compared to the day with no intervention and Subject D only showed a marginal $1 \%$ improvement. Our brightness intervention showed the most significant improvement of each intervention type with all subjects showing an increase of more that $30 \%$ of their time being spent in good posture.

\subsection{Periods of Poor Posture}

Next we analysed our data to find the longest period of continuously poor posture for each subject, again divided out by intervention type as shown in Figure 3. From this data we can see that two of our subjects had their longest period of poor posture on the day with no interventions. Our motivational intervention resulted in improvement for only two out of the four subjects, with subjects $\mathrm{C}$ and D showing longer times spend sitting in poor postures. 
Our information-based intervention performed better with three of our subjects showing an improvement, however subject $\mathrm{C}$ spent more than three times longer sitting continuously in poor posture than they had with no intervention at all. The brightness intervention showed the most notable effect on the time spent sitting continuously with poor posture. All of our subjects showed a decrease in the number of consecutive minutes spent sitting with poor posture.

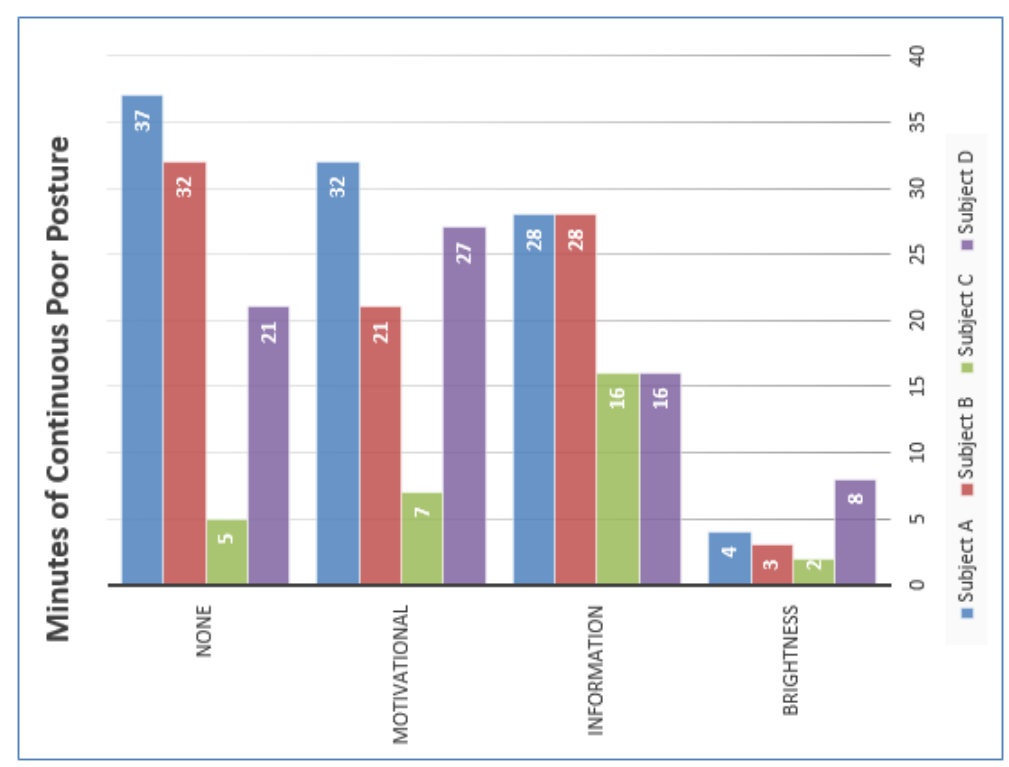

Fig. 3. Longest continuous period of poor posture by subject and intervention type.

\subsection{Posture Transitions}

Finally we extracted the number of times our subjects posture shifted from good to poor and back again. From Figure 4 we can see that the number of transitions per subject when no intervention was delivered was quite widely dispersed, ranging from 110 to 375 transitions in a period of four hours. This may indicate the different sitting habits of each of our subjects. Data for our motivational intervention shows that the number of transitions decreased for two subjects which may indicate a heightened awareness of their posture, however the number of transitions increased for the other two subjects. Likewise, our information intervention showed improvement in two subjects and dis-improvement in the other two. Our brightness intervention is the only intervention to provide a decrease in the number of posture transitions for all four subjects.

This is interesting as it indicates that subjects maintained a more constant posture throughout the experiment when receiving the brightness intervention. 


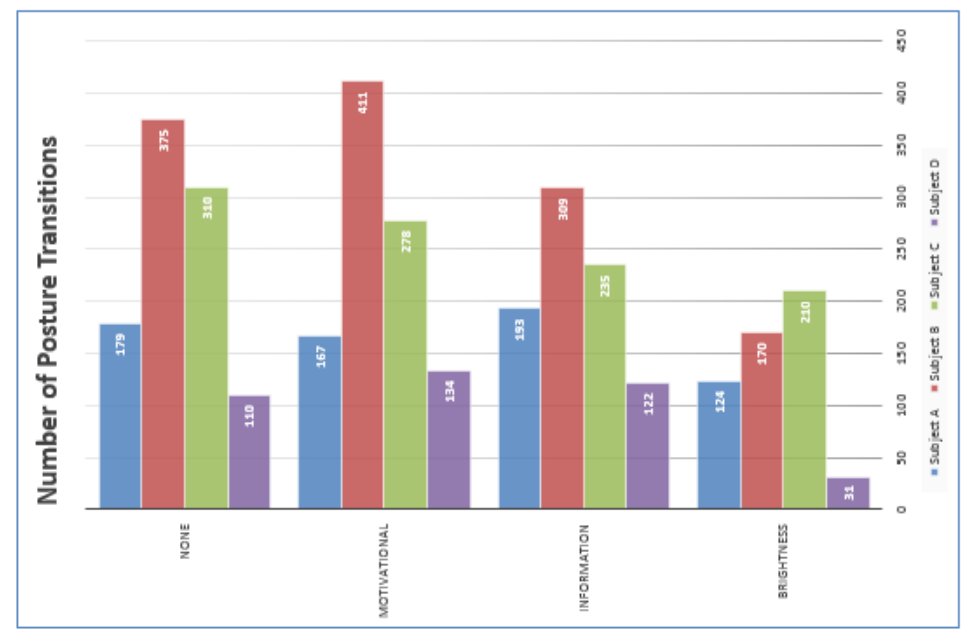

Fig. 4. Total number of posture transitions.

Our brightness intervention is designed to immediately correct the subject's posture when it moves out of a good position, and as such it could have been expected that subjects would have a higher number of transitions due to the number of interventions. Instead we see that subjects have fewer transitions, meaning they are more aware of what their good posture position is and they are better able to maintain it.

We also noted some comments from the subjects in our trial, all four subjects remarked that the brightness intervention was the most intuitive and found they were more conscious of their posture during that day's session. One subject noted that simply the presence of the sensor pointed at them made them more aware of their posture during the trials. Another subject noted that they forgot about the presence of the sensor until they received interventions on days with the information and motivational interventions.

\section{Conclusions}

From our results we can see that the most successful intervention type was that based on monitor brightness. This proves our original hypothesis that an intervention based around an immersive subject experience will be the most effective way to correct a person's posture. While the motivational and information based interventions provided some improvements to our subject's posture the degree of improvement was significantly less than with our monitor brightness intervention. We can also conclude that the Kinect ${ }^{\mathrm{TM}}$ can be used to effectively monitor a person's posture in an office environment and can do so without the need for image or video capturing of the subject. This is vital for providing a posture intervention system that protects the person's privacy. 
In terms of future work, there are questions that remain to be investigated. Our study was limited to subjects using desktop PCs in an office environment and required the sensor to be mounted on a stand behind the subject's computer screen. We would like to explore sensors embedded in a monitor to provide the same intervention experience with a less intrusive presence in the working environment. Since our software only used a portion of the Kinect's ${ }^{\mathrm{TM}}$ capability, we could design an ever lower cost sensor for the purpose of posture classification.

Since our study was limited to four days per subject, the effects of each intervention type over a prolonged period of time could not be investigated. It would be interesting to see if the information or motivational interventions have a stronger effect on subjects after a longer period. Finally, the question of how willing people are to subject themselves to these types of interventions with the purpose of improving their posture has not been explored. Neither has the impact of these interventions of a person's productivity or concentration while carrying out work on a computer. Once again, these are for further study.

Acknowledgements This research was supported by Science Foundation Ireland SFI/12/RC/2289.

\section{References}

1. Che-hsu Joe Chang, Benjamin C Amick, Cammie Chaumont Menendez, Jeffrey N Katz, Peter W Johnson, Michelle Robertson, and Jack Tigh Dennerlein. Daily computer usage correlated with undergraduate students' musculoskeletal symptoms. American journal of industrial medicine, 50(6):481-488, 2007.

2. Ross A Clark, Yong-Hao Pua, Karine Fortin, Callan Ritchie, Kate E Webster, Linda Denehy, and Adam L Bryant. Validity of the microsoft kinect for assessment of postural control. Gait \& P Posture, 36(3):372-377, 2012.

3. Shona Fang, Jonathan Dropkin, Robin Herbert, Dushana Triola, and Paul Landsbergis. Workers' compensation experiences of computer users with musculoskeletal disorders. American journal of industrial medicine, 50(7):512-518, 2007.

4. BJ Fogg. Creating persuasive technologies: an eight-step design process. In Persuasive, page 44, 2009.

5. Brian J Fogg. Persuasive computers: perspectives and research directions. In Proceedings of the SIGCHI conference on Human factors in computing systems, pages 225-232. ACM Press/Addison-Wesley Publishing Co., 1998.

6. Fred Gerr, Michele Marcus, and Carolyn Monteilh. Epidemiology of musculoskeletal disorders among computer users: lesson learned from the role of posture and keyboard use. Journal of Electromyography and Kinesiology, 14(1):25-31, 2004.

7. Michael Haller, Christoph Richter, Peter Brandl, Sabine Gross, Gerold Schossleitner, Andreas Schrempf, Hideaki Nii, Maki Sugimoto, and Masahiko Inami. Finding the right way for interrupting people improving their sitting posture. In HumanComputer Interaction-INTERACT 2011, pages 1-17. Springer, 2011.

8. Alejandro Jaimes. Sit straight (and tell me what i did today): a human posture alarm and activity summarization system. In Proceedings of the 2nd ACM workshop on Continuous archival and retrieval of personal experiences, pages 23-34. ACM, 2005.

9. Brian D Lowe. Accuracy and validity of observational estimates of shoulder and elbow posture. Applied ergonomics, 35(2):159-171, 2004. 